\title{
Inhaled resveratrol treatments slow ageing-related degenerative changes in mouse lung
}

\author{
Sonia Navarro, ${ }^{1,2}$ Raghava Reddy, ${ }^{1}$ Jooeun Lee, ${ }^{1}$ David Warburton, ${ }^{1}$ Barbara Driscoll ${ }^{1}$
}

- Additional material is published online only. To view please visit the journal online (http://dx.doi.org/10.1136/ thoraxjnl-2016-208964).

'Developmental Biology and Regenerative Medicine, Saban Research Institute, Children's Hospital Los Angeles, Los Angeles, California, USA ${ }^{2}$ Craniofacial Biology Graduate Program, Herman Ostrow School of Dentistry of University of Southern California, Los Angeles, California, USA

\section{Correspondence to}

Professor Barbara Driscoll, Developmental Biology and Regenerative Medicine, Saban Research Institute, Children's Hospital Los Angeles, 4650

Sunset Blvd, Los Angeles, CA 90027,USA; bdriscoll@chla. usc.edu

Received 25 May 2016 Revised 4 November 2016 Accepted 28 November 2016 Published Online First 19 January 2017

\section{ABSTRACT}

Background Lung ageing, a significant risk factor for chronic human lung diseases such as COPD and emphysema, is characterised by airspace enlargement and decreasing lung function. Likewise, in prematurely ageing telomerase null (terc-/-) mice, p53 stabilisation within diminishing numbers of alveolar epithelial type 2 cells (AEC2) accompanies reduced lung function. Resveratrol (RSL) is a plant phytoalexin that has previously showed efficacy in enhancing invertebrate longevity and supporting mammalian muscle metabolism when delivered orally. Here, we tested whether inhaled RSL could protect young, terc-/- mice from accelerated ageing of the lung.

Methods terc $-1-$ mice aged 2 months inhaled $1 \mathrm{mg} /$ $\mathrm{kg}$ RSL that was instilled intratracheally once per month for 3 months. One month after the last inhalation, whole lung function, structure and cellular DNA damage were evaluated and AEC2 survival was assessed by western blotting for survival pathway gene expression.

Results RSL treatments delayed the loss of lung compliance $(p<0.05)$, maintained lung structure $(p<0.001)$ and blocked parenchymal cell DNA damage as measured by TdT Nick-End Labeling (TUNEL). RSL, a known agonist of deacetylase SIRT1, supported AEC2 survival by stimulating SIRT1 expression, promoting p53 destabilisation and decreasing Bax expression and by maintaining expression levels of Peroxisome proliferatoractivated receptor gamma coactivator 1 -alpha (PGC-1 $\alpha$ ), activated p-Akt and p-Mdm2 and inactivated PhosphoPhosphatase and tensin homolog ( $p$-PTEN).

Conclusions RSL prophylaxis by inhalation is a potential approach for slowing ageing-related deterioration of lung function and structure by maintaining $A E C 2$ integrity.

\section{INTRODUCTION}

In healthy young adults, breathing is an essential, efficient process, but natural ageing of the lung then occurs at a steady and irreversible rate, as measured by an inexorable decline in lung function. ${ }^{1-3}$ Altered properties of lung structure and compromise of cell populations due to wear, telomere shortening, increased senescence and regenerative stem cell failure drive this process. ${ }^{4-7}$ Thus, natural deterioration leads to a significantly reduced quality of life, over a time frame dependent on genetic and environmental factors. Although some available therapies can ameliorate symptoms, ageing-related lung failure is generally irreversible and is accompanied by high rates of morbidity and mortality due to increased disease risk, including
Key messages

What is the key question?

- Can the accumulation of ageing-related degenerative changes in the lung be slowed by inhaled, prophylactic treatments?

What is the bottom line?

- Inhaled, prophylactic resveratrol treatments can slow the rate of the lung function decline, alveolar enlargement and alveolar epithelial type 2 cell DNA damage that occurs in the early stages of lung ageing.

\section{Why read on?}

- Administration of resveratrol directly to the lungs may be an effective intervention for lung ageing, which is a major risk factor for development of chronic lung disease.

development of COPD, with accompanying emphysema and chronic bronchitis. ${ }^{8}$

Mouse models are becoming increasingly informative for determining specific mechanisms that contribute to irreversible lung ageing. ${ }^{9}$ The terc-/mouse is a serviceable model of accelerated ageing. ${ }^{10}$ In humans, telomere shortening has been linked to development of lung fibrosis and emphysema. ${ }^{11}$ Our laboratory has previously shown that terc-/- mice also exhibit several hallmarks of ageing in both whole lung and in alveolar epithelial type 2 cells (AEC2), which have been identified as stem cells of the adult lung alveoli. ${ }^{12}$ We showed that whole lung structure, collagen deposition, AEC2 integrity and the ability to survive and regenerate lung tissue following partial pneumonectomy are compromised in the terc-/- model, in a way tightly correlated to telomere length. ${ }^{13}{ }^{14}$ Armanios and Hogan recently showed that telomere dysfunction, specifically in AEC2, causes whole organ vulnerability to lung injury. ${ }^{15}$ Taken together, these studies show that the AEC2 population is both a marker of, and a critical target for, lung ageing. Despite evidence that shows AEC2 integrity plays a significant role in maintaining lung health and homeostasis, there is currently little understanding of how to inhibit loss of AEC2 or replenish the population, or what impact AEC2 preservation might have on whole lung ageing.

Resveratrol (RSL) is an agonist of sirtuin deacetylase SIRT1. ${ }^{16}{ }^{17}$ Sirtuins are master regulators of 
metabolism with multiple targets. ${ }^{18} 19$ SIRT1 deacetylates Trp53, which destabilises it and leads to cell cycle entry and inhibition of apoptosis. $^{20} 21$ The role of Trp53 stabilisation in increased lung disease risk and decreased ability to respond to injury has recently been recognised by our lab and others. ${ }^{11} 131422$ SIRT1 also activates PGC-1 $\alpha$, a master regulator of mitochondrial biogenesis. ${ }^{23}{ }^{24}$ SIRT1 expression and activity decrease with age and the onset of ageing-related diseases, including COPD. ${ }^{8} 1625$ While RSL treatment effectively extends lifespan in lower organisms, ${ }^{26}$ this result has not been directly duplicated in mice or humans. ${ }^{27}{ }^{28}$ Controversies regarding effective RSL dose, administration strategies and efficacy still exist. However, there current data indicate that RSL may extend the mammalian healthspan by supporting the efficient function of critical organs and their progenitor populations over the lifetime of the organism, potentially including the lung. ${ }^{29-32}$

To determine whether prophylactic RSL inhalational treatments of terc- $/-$ mice aged $2-4$ months from the second filial (F2) generation (terc-/-F2) could preserve youthful whole lung function and AEC2 survival, cohorts aged 2 months received either RSL or vehicle by intratracheal (IT) instillation once per month for 3 months. One month following the final treatment, whole lung function was assessed by plethysmography and whole lung tissue and AEC2 were harvested and analysed. We found that RSL treatments were effective in slowing ageing-related degeneration of whole lung and AEC2. We believe that ours is the first study to demonstrate a beneficial effect of lung-directed RSL treatments on ageing lung function and structure.

\section{METHODS}

Method details on lung function measurements, histology, mean linear intercept (MLI) analysis, TUNEL in situ, collagen and elastin quantitation, mitochondrial bioenergetics assays and western blotting can be found in the online supplementary material methods section.

\section{Animal experiments}

All animal studies were performed in accordance with The Guide, American College of Laboratory Animal Medicine (ACLAM) guidelines and Animal Research: Reporting of In Vivo Experiments (ARRIVE) guidelines at Children's Hospital Los Angeles/The Saban Research Institute (CHLA/TSRI), an Association for Assessment and Accreditation of Laboratory Animal Care (AAALAC)-accredited institution. Studies were submitted to, and approved by, the Institutional Animal Care and Use Committee of CHLA/TSRI prior to commencing work. Wild-type (WT) control and experimental terc-/-F2 mice $\left(\mathrm{C} 57 \mathrm{Bl} / 6 \mathrm{~J}\right.$ and $\mathrm{B} 6 . \mathrm{Cg}-\operatorname{Terc}^{\text {tm } 1 R d p} / \mathrm{J}$ respectively, bred at the CHLA/TSRI Animal Care Facility (ACF), a specific pathogenfree facility, where mice were housed under standard conditions as per The Guide), weight 20-25 g, were randomised for gender into cohorts of 6-10 for each experiment. All terc-/-F2 treatment groups received $1 \mathrm{mg} / \mathrm{kg}$ RSL (Sigma) in $20 \%$ dimethyl sulfoxide (DMSO) in phosphate buffered saline (PBS) (RSL reconstituted in 100\% DMSO was diluted 1:5 in PBS to produce the required concentration) via IT administration to directly target lungs at 2, 3 and 4 months of age. All terc-/-F2 vehicle groups received 20\% DMSO in PBS only. Additional treatments included, $1 \mathrm{mg} / \mathrm{kg}$ Ex527 alone or in combination with $1 \mathrm{mg} / \mathrm{kg}$ RSL. On the day of IT delivery, the cohort that received vehicle only was treated first, followed by all mice that received RSL. IT delivery of Ex527, alone or in combination with RSL, was performed on a separate day. IT delivery was performed under isoflurane anaesthesia in laminar flow hoods within the CHLA/TSRI ACF. Administrations occurred once a day at mid-morning. Animals were humanely euthanised and removed from cohort analysis in the rare instances that IT administration failed. Otherwise mice remained in the study housed under standard conditions and were euthanised at age 5 months, 1 month after the last IT administration. For a number of experiments, whole lung function was assessed and tissue was analysed from untreated controls (both aged 2 and 5 months).

\section{Statistics}

Data were analysed with GraphPad Software one-way analysis of variance and Bonferroni post hoc analysis was used for multiple comparison of the means.

\section{RESULTS}

\section{RSL inhalation partially preserves lung function}

To examine the impact of IT instillations, pulmonary function testing was performed and tissue was harvested from duplicate cohorts of RSL-treated and vehicle-administered mice 1 month after the final treatment (figure 1). To determine baseline lung function in ageing mice as measured by dynamic compliance (defined as a function of lung stiffness), lung mechanics were performed on WT $(\mathrm{C} 57 \mathrm{Bl} / 6 \mathrm{~J}$, the background strain for the terc-/- mutants) and terc-/-F2 mice (figure 2A). As expected in a rapidly ageing animal, at 5 months of age without treatment terc-/-F2 lung tissue compliance was significantly increased versus mice aged 2 months, demonstrating the degree and rapidity of loss of lung function in the terc-/-F2 model. Results for untreated terc-/-F2 mice at 5 months were consistent with a loss of elasticity and increased air spaces, as we previously described for terc-/- mice. ${ }^{14}$ There was also an increase in compliance in untreated WT cohorts at 5 months, but the degree of increase, while significant, was much lower. Compliance for young terc-/-F2 mice aged 2 months did not significantly differ from that of WT mice aged 2 months.

Lung mechanics were then measured 1 month following the third and last RSL treatment or delivery of vehicle to terc-/-F2

\section{Treatment: PBS or $1 \mathrm{mg} / \mathrm{kg}$ Resveratrol of terc-/-F2 mice only}

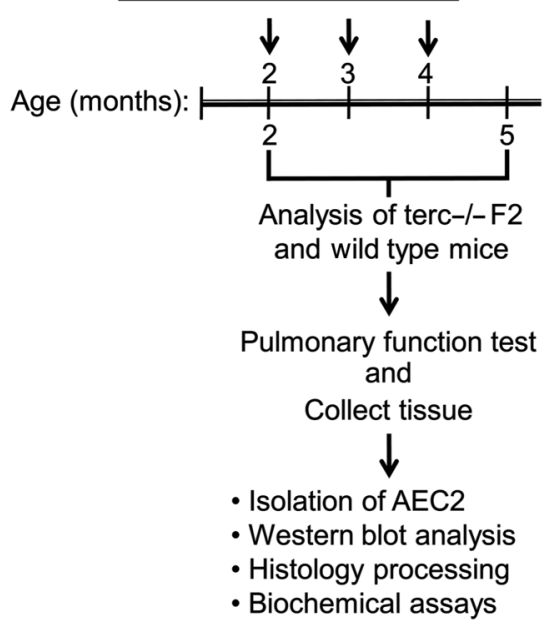

Figure 1 Intratracheal prophylactic administration of resveratrol. Experimental protocol for evaluation of resveratrol impact on ageing terc-/-F2 mice. AEC2, alveolar epithelial type 2 cells; PBS, phosphate buffered saline. 
A

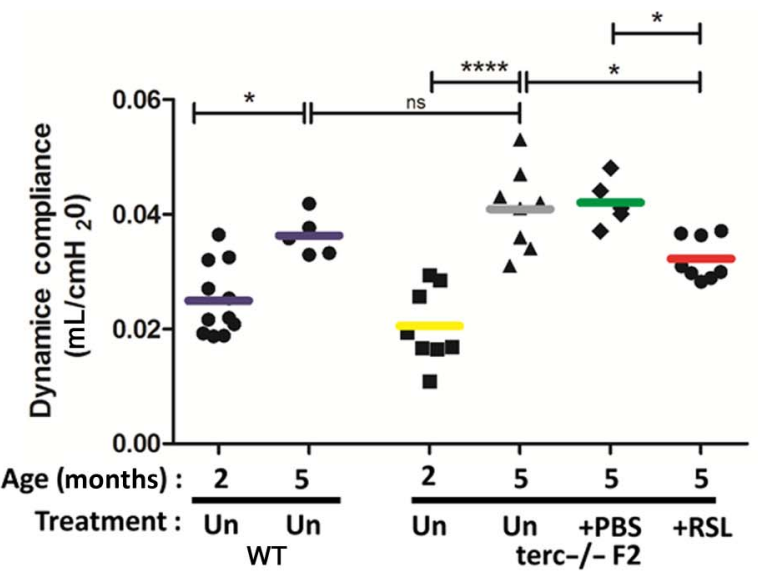

B

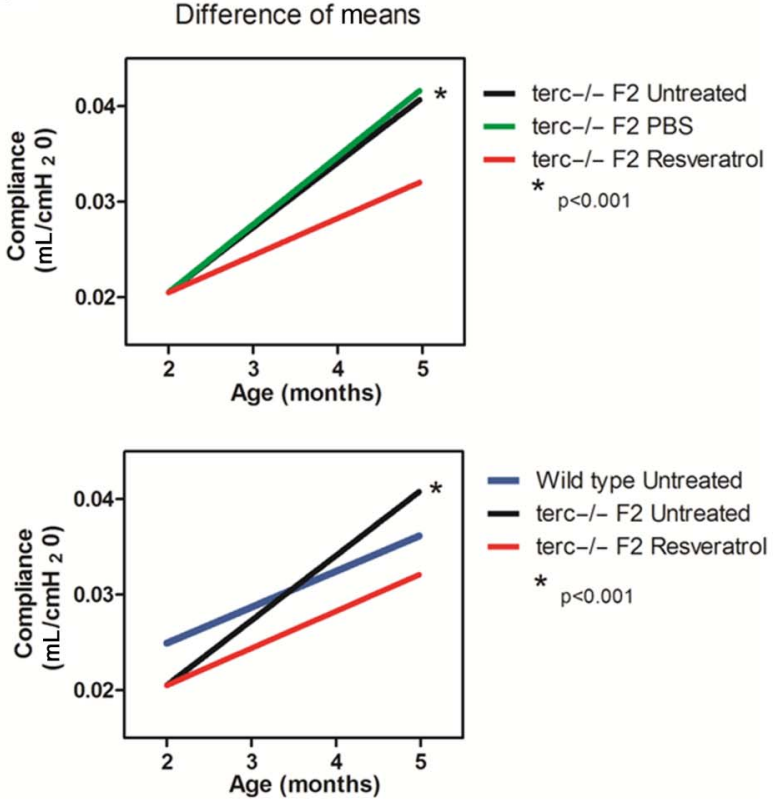

Figure 2 Dynamic lung compliance following resveratrol (RSL) treatment. (A) Baseline lung function compliance for both the wild-type (WT) and terc-I-F2 mice was measured at 2 and 5 months of age without treatment (Un). Each data point represents a single animal, with the value calculated as an average of triplicate measurements. The bar for each cohort represents the group mean. As expected, compliance increased in both cohorts, but increased much more rapidly in terc $-I-F 2$ lung $\left({ }^{*} p<0.05\right.$ vs $\left.{ }^{* *} p<0.001\right)$. For treatment studies, terc $-I-F 2$ mice aged 2 months received three doses of $1 \mathrm{mg} / \mathrm{kg}$ RSL or vehicle control (phosphate buffered saline (PBS)) at monthly intervals. At 5 months of age, mice that received only PBS showed no significant changes in compliance and were comparable to the untreated cohort aged 5 months. However, compliance was significantly lower in lungs of RSL-treated mice compared with untreated and PBS-treated control mice ( ${ }^{*} \mathrm{p}<0.05$; untreated terc $-I-$ aged 2 months $\mathrm{N}=8$; untreated terc $-I-$ aged 5 months $\mathrm{N}=8, \mathrm{PBSN}=5, \mathrm{RSL} N=8$ ). There was no significant difference between the WT and terc $-I-\mathrm{F} 2$ mice aged 5 months. (B) Calculation of the change in mean compliance over time in untreated terc-I-F2 mice or those that received PBS or RSL treatment showed an $83 \%$ increase in untreated and vehicle-treated cohorts compared with the RSL-treated group $\left(r^{2}=0.71, r^{2}=0.78\right.$ and $r^{2}=0.56$, respectively). $R^{2}$ value was not significantly different when changes in mean compliance over time were compared between RSL-treated terc-I-F2 and WT controls $\left(r^{2}=0.56\right.$ and $\left.r^{2}=0.49, p=0.87\right)$. Statistical analysis was performed by one-way analysis of variance and Bonferroni multiple comparison test, and linear regression analysis was used to compare slopes.

mice. Mice that received only vehicle showed no significant changes in compliance compared with untreated mice aged 5 months. However, after RSL treatments, lung compliance was significantly lower than that of untreated controls (by 21\%; $\mathrm{p}<0.05$ ), suggesting partial maintenance of youthful lung elastic recoil (figure $2 \mathrm{~A}$ ).

We also analysed the rate of compliance loss for all cohorts in order to determine the difference between deterioration in RSL-treated versus untreated terc-/-F2 cohorts, as well as the similarities in compliance maintenance between RSL-treated terc-/-F2 and untreated WT cohorts (figure 2B). The compliance rate change of lungs treated with RSL was moderate compared with the steeper slope of terc-/-F2 untreated or PBS-treated cohorts and was comparable to that of untreated WT cohorts. This analysis showed that the change in compliance of the respiratory system treated with RSL follows a similar, slower pattern, similar to that of healthy, WT lungs.

\section{RSL inhalation preserves lung parenchymal structure, but does not affect the extracellular matrix}

To evaluate changes in lung parenchyma in untreated terc-/-F2 mouse lung versus lung structure following administration of RSL, air spaces were quantified using MLI analysis (figure 3A). As previously described, ${ }^{14}$ tissue samples that featured large airways and blood vessels were excluded in the analysis of parenchymal tissue photomicrographs (figure $3 \mathrm{~B}$ ). WT mice at matched ages were used as controls. In WT lung, differences in MLI were observed between mice aged 2 and 5 months but were not statistically significant. In contrast, statistically significant changes in MLI were detected in control terc-/-F2 mice aged 5 months compared with terc-/- mice aged 2 months. This alteration in distal lung structure was consistent with our previously published study on changes in terc-/- lung. ${ }^{14}$ Following RSL treatment, this loss of structure appeared to be nullified, as lung morphometry measurements of treated terc $-/-F 2$ lungs exhibited an approximately 20\% lower MLI value $(28.0 \pm 1.3 \mu \mathrm{m})$ compared with lung tissue from mice that received vehicle $(35.0 \pm 1.9 \mu \mathrm{m})$ and $17 \%$ lower when compared with tissue from untreated mice $(33.6 \pm 1.1 \mu \mathrm{m})$. There was no significant difference in MLI between the vehicle-administered and untreated terc-/-F2 mice. Thus, MLI analysis showed a significant impact by prophylactic RSL treatments on age-related deterioration of lung structure.

To determine if lung extracellular matrix (ECM), which provides structure to the lung and supports its function, was affected by RSL treatment of terc-/-F2 mice, whole lung collagen and elastin content were quantitated as described in online supplementary material results and shown in online supplementary figure $\mathrm{S} 1$. We did not observe substantial changes in ECM maintenance in RSL-treated mice, although we also noted that the ECM compartment was not adversely affected at this early stage of ageing in terc-/-F2 lung. These data indicated that at 5 months of age, lung components other than the ECM were responsible for the altered pulmonary function and structure observed in early ageing, and that the ECM was not a primary target for early stage RSL prophylaxis. 


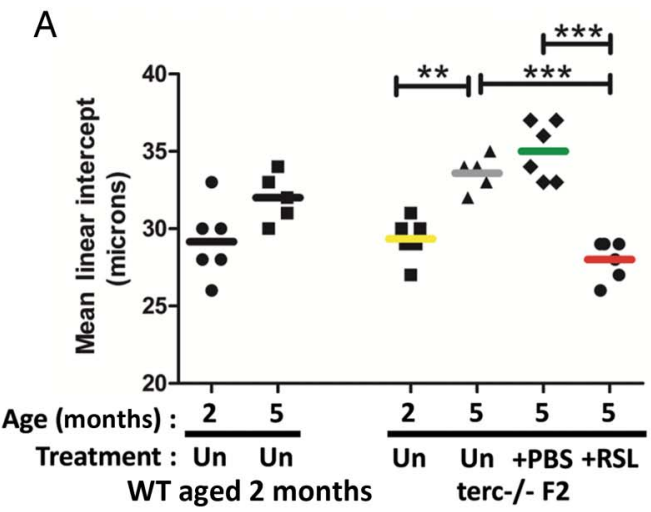

B
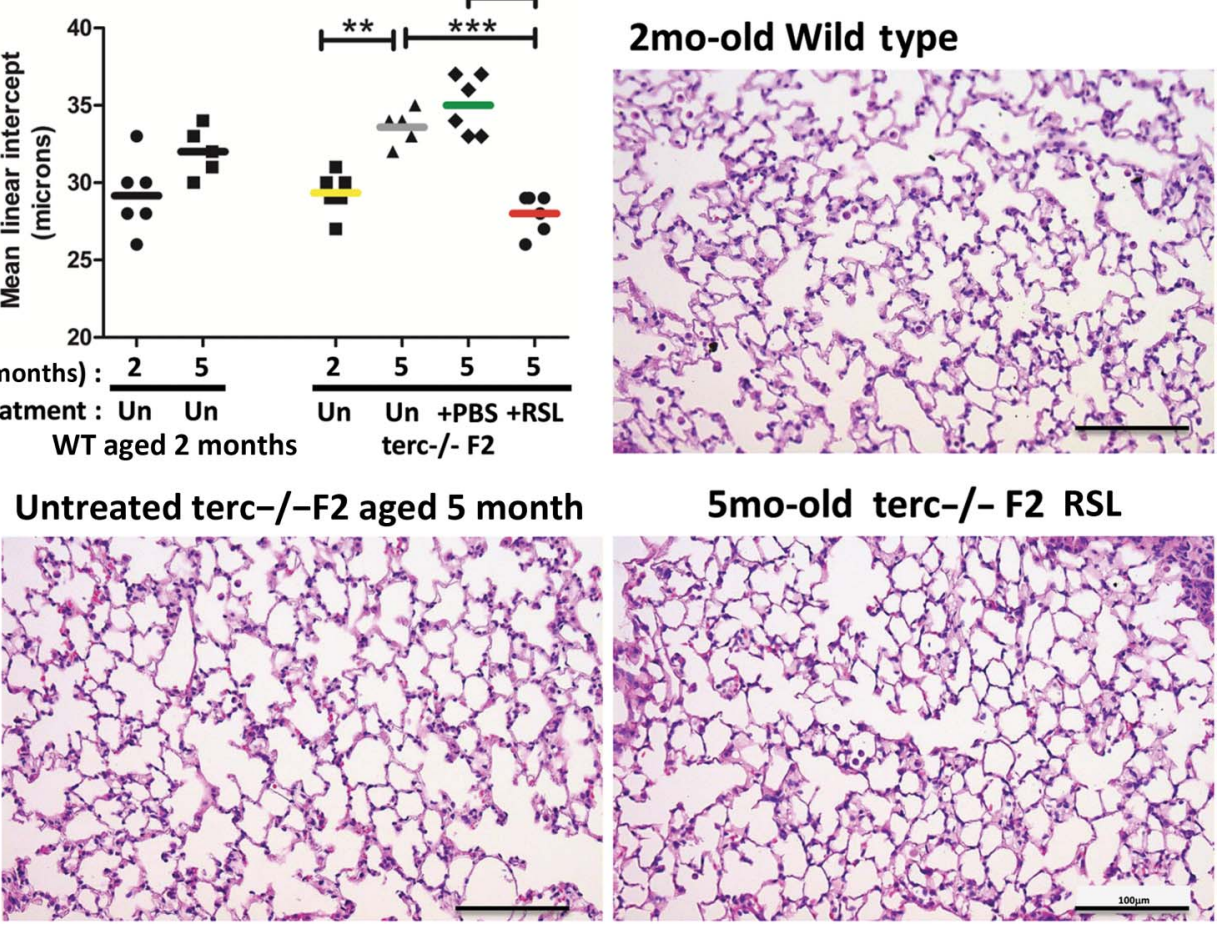

Figure 3 Lung structure following resveratrol (RSL) treatment. (A) Quantification of mean linear intercept (MLI) in RSL-treated versus phosphate buffered saline (PBS) administered or untreated terc-I-F2 mice 1 month after the last treatment. RSL-treated cohorts showed a significantly $\left({ }^{* *} p<0.001\right)$ lower MLI than PBS and untreated mice. Wild type (WT) mice aged 2 and 5 months were also analysed for comparison with no significant difference, while the terc-l-F2 mice of the same age range showed a significant $\left({ }^{* *} p<0.01\right)$ increase in MLI. Each data point represents a single animal, with the value calculated as an average of triplicate measurements. The bar for each cohort represents the group mean (terc-I-F2 untreated aged 2 months $\mathrm{N}=6$; terc-I-F2 5 months untreated $\mathrm{N}=5$; PBS $\mathrm{N}=6$; RSL $\mathrm{N}=6$; WT aged 2 months $\mathrm{N}=6$; WT aged 5 months $\mathrm{N}=5$ ). Statistical analysis was performed by one-way analysis of variance and the Bonferroni multiple comparison test. (B) Representative distal lung sections, absent large airways and vessels, which were used for MLI analysis stained with H\&E. Scale bar for $20 x$ images is 100 microns.

\section{RSL inhalation stimulates SIRT1 and maintains youthful levels of PGC-1 $\alpha$ in AEC2, but does not affect AEC2 mitochondrial bioenergetics}

Given the apparent lack of ECM targeting by RSL treatments, we next investigated whether RSL treatments maintained ageing lung structure and function by targeting pathways that control AEC2 mitochondrial function. As in other cell types, accelerated ageing in the lung could correlate with a decline in respiratory chain function, which is associated with ageing, ${ }^{33} 34$ but the inter-relationship between lung function and AEC2 mitochondrial function is not well understood. By western blotting of isolated terc-/-F2 AEC2 and AEC2 from WT mice, we found that RSL treatments moderately stimulated levels of SIRT1 by fourfold, while levels of PGC- $1 \alpha$, an endogenous substrate of SIRT1, were maintained at youthful levels (figure 4A, B). Collectively, these data indicated that prophylactic, intermittent, inhalation of RSL was capable of activating SIRT1 in AEC2 in vivo, while also maintaining PGC- $1 \alpha$ levels.

We next investigated AEC2 mitochondrial bioenergetics using the Seahorse assay, which measures oxygen consumption rate (OCR) in vitro, as described in online supplementary material results and shown in online supplementary figure S2A. This analysis showed a significant decrease in terc-/-F2 AEC2 bioenergetics at 16 months of age, but no significant change in bioenergetics when OCR from WT and terc-/-F2 AEC2 mice aged 2-3 months were compared, and no improvement in mitochondrial OCR in AEC2 isolated from RSL-treated mice aged 5 months (see online supplementary figure S2B). Thus, despite maintenance of PGC- $1 \alpha$ expression in AEC2 isolated from RSL-treated lungs, the impact on mitochondrial function at this early stage of lung ageing was negligible. We concluded that lower mitochondrial bioenergetics due to decreasing levels of PGC-1 $\alpha$ will cause a slowdown in mitochondrial biogenesis in terc-/-F2 mice at a later stage of lung ageing, but that this impact does not become significant until animals are considerably older than 5 months.

\section{RSL prophylaxis blocks AEC2 DNA damage and destabilises Trp53}

We next investigated whether inhaled RSL contributed to lung structure and function maintenance by blocking ageing-related alveolar epithelial DNA damage. To do this, TUNEL stained lung sections from untreated versus RSL-treated, vehicleadministered, Ex527 (a SIRT1 inhibitor) or Ex527 plus RSL terc -/-F2 mice were analysed by manual counting for TUNEL, measuring fluorescently labelled single-strand breaks in DNA. As has previously been reported by ourselves and others, lungs from healthy WT controls aged 2 months show very few TUNELpositive cells (not shown), while lungs from terc-/-F2 mice aged 2 months exhibit substantial numbers of TUNEL-positive cells. Untreated terc-/-F2 controls at age 5 months had double the number of TUNEL-positive cells compared with untreated controls aged 2 months, while the number of TUNEL-positive, single-stranded DNA breaks in alveolar epithelium from RSL-treated lungs were significantly fewer, to the point where the increase at 5 months of age was nullified (figure 5A, B). 
A
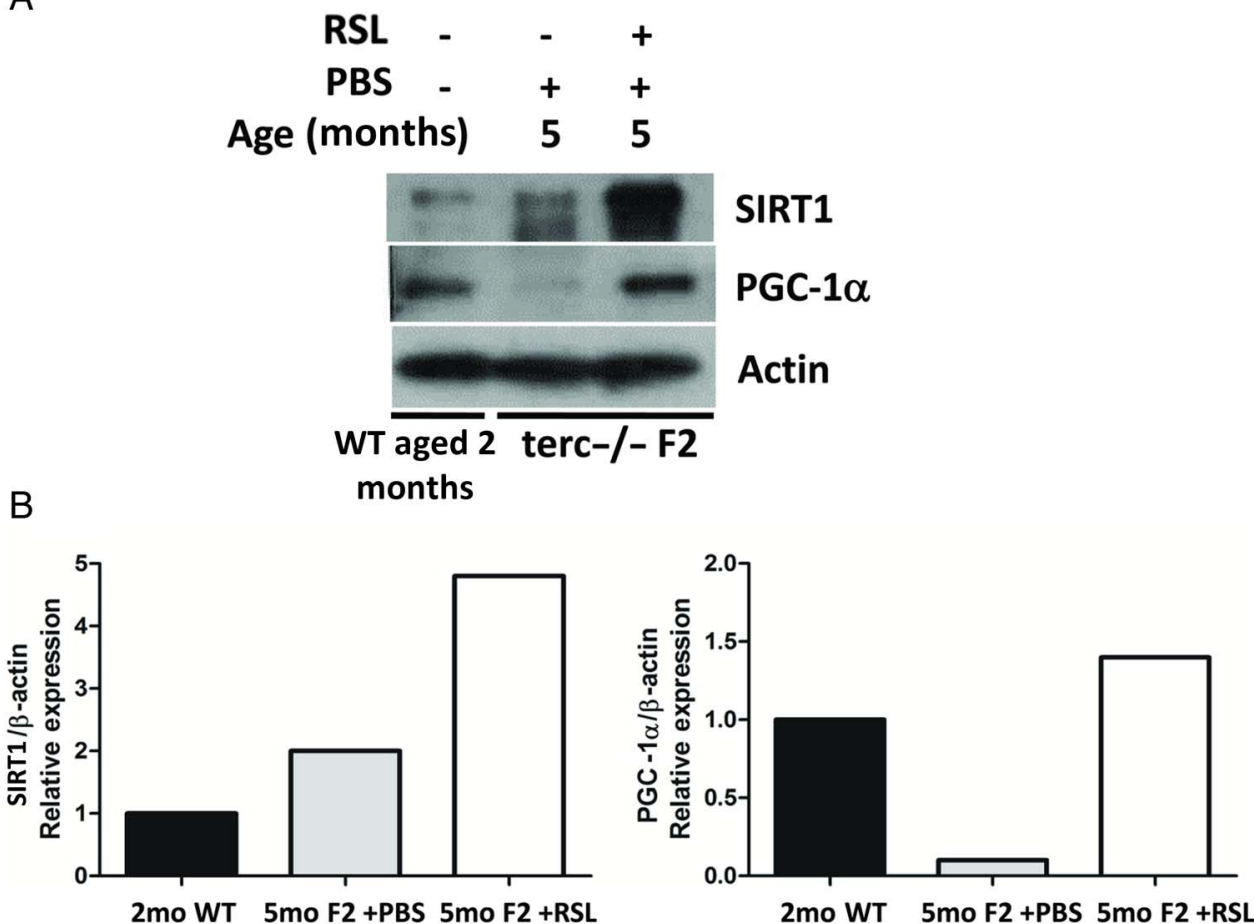

Figure 4 SIRT1 and PGC-1 $\alpha$ expression in alveolar epithelial type 2 cells (AEC2) following resveratrol (RSL) treatment. (A) Immunoblot analysis showed increased expression SIRT1 by fourfold in AEC2 from RSL-treated mice versus levels in AEC2 from age-matched vehicle-administered controls. Analysis also showed maintenance of PGC-1 $\alpha$ in AEC2 from RSL-treated lung at levels similar to that in AEC2 isolated from untreated wild type (WT) at 2 months. In contrast, PGC-1 $\alpha$ levels in AEC2 from mice that received vehicle only were considerably lower at the 5-month time point. (B) Scanning densitometry showed that the increase in SIRT1 expression in AEC2 isolated from RSL-treated samples was fourfold compared with WT samples aged 2 months. PGC-1 $\alpha$ expression in RSL treated samples aged 5 months was comparable to levels in AEC2 isolated from untreated mice aged 2 months. Blots are representative of, at minimum, two blots. Each sample was composed of lysates equally pooled from three mice.

Moreover, SIRT1 inhibition by Ex527 or in combination with RSL showed a significant increase in TUNEL-positive cells. Surfactant protein C (SPC)-positive cell counts (figure 5C) further confirmed the preservation of the AEC2 population following RSL treatments, which were absent in all other treated groups. These studies produced direct evidence that inhaled RSL has the potential to restrict DNA damage that could eventually lead to apoptosis of rapidly ageing alveolar epithelium in vivo.

To determine a potential mechanism for reduction of DNA damaged alveolar epithelium by inhaled RSL, which presumably included reduced numbers of damaged AEC2, we examined the protein expression levels of pro-apoptotic protein $\operatorname{Trp5} 3$ in freshly isolated AEC2. Decreased expression of activated pSer15-p53 was detected in AEC2 from RSL-treated mice aged 5 months when compared with levels in PBS-administered controls by western blotting (figure $5 \mathrm{D}, \mathrm{E}$ ). These data indicate that inhaled RSL can act on one of its known targets, $\operatorname{Trp} 53$, to cause degradation of the stabilised form of the protein, which in turn induces more rapid Trp53 turnover, thus enhancing cell survival and suppressing AEC2 apoptosis.

\section{RSL prophylaxis preserves AEC2 survival pathway protein expression}

To determine if other anti-apoptotic, prosurvival pathways were maintained in terc-/-F2 AEC2 by RSL treatments, we also analysed components of various cell survival pathways by western blotting (figure 6). We found that inhaled RSL blocked rising pro-apoptotic Bax levels and maintained levels of Trp53-binding protein $\mathrm{Mdm} 2$ in ageing terc-/-F2 AEC2. These data correlated with our observations that $\operatorname{Trp} 53$ expression levels were reduced in these same cells. In addition, we noted that the activated, phosphorylated form of survival promoter Akt and the deactivated, phosphorylated form of the growth suppressor, PTEN, were also maintained at youthful levels in AEC2 isolated from RSL-treated lungs. These data indicated that the pathways that are active in youthful WT AEC2 aged 2 months, which decline rapidly in ageing, terc null cells, can successfully be maintained by prophylactic administration of intermittent, inhaled RSL.

\section{DISCUSSION}

Ageing, with accompanying telomere shortening, is a major risk factor for chronic lung disease. ${ }^{6}{ }^{11}$ This risk is presumed in part to be based on the gradual breakdown of structural support and diminished function of the AEC2 stem cell population, although many studies have also shown that ageing, as a global phenomenon in the lung, also impacts resident endothelial, mesenchymal and immune cell populations. ${ }^{14} 15$ These ageing-related changes lead to a decline in the ability of the lung to respond efficiently to insults ${ }^{13}$ and repair damage from exposure to environmental stressors. While the AEC2 population may retain some capacity for plasticity and robustness over lifespan, mechanisms for supporting this critical population, which serves as both a distal lung stem cell and a source of surfactant, are still not well understood.

Our data now show that RSL prophylaxis has a positive impact on at least one critical cellular component of the lung, $\mathrm{AEC} 2$, as well as on whole lung function and structure in terc $-/-F 2$ mice aged 5 months. Data further indicate that the main target of RSL is not the ECM or the mesenchymal/immune cells 


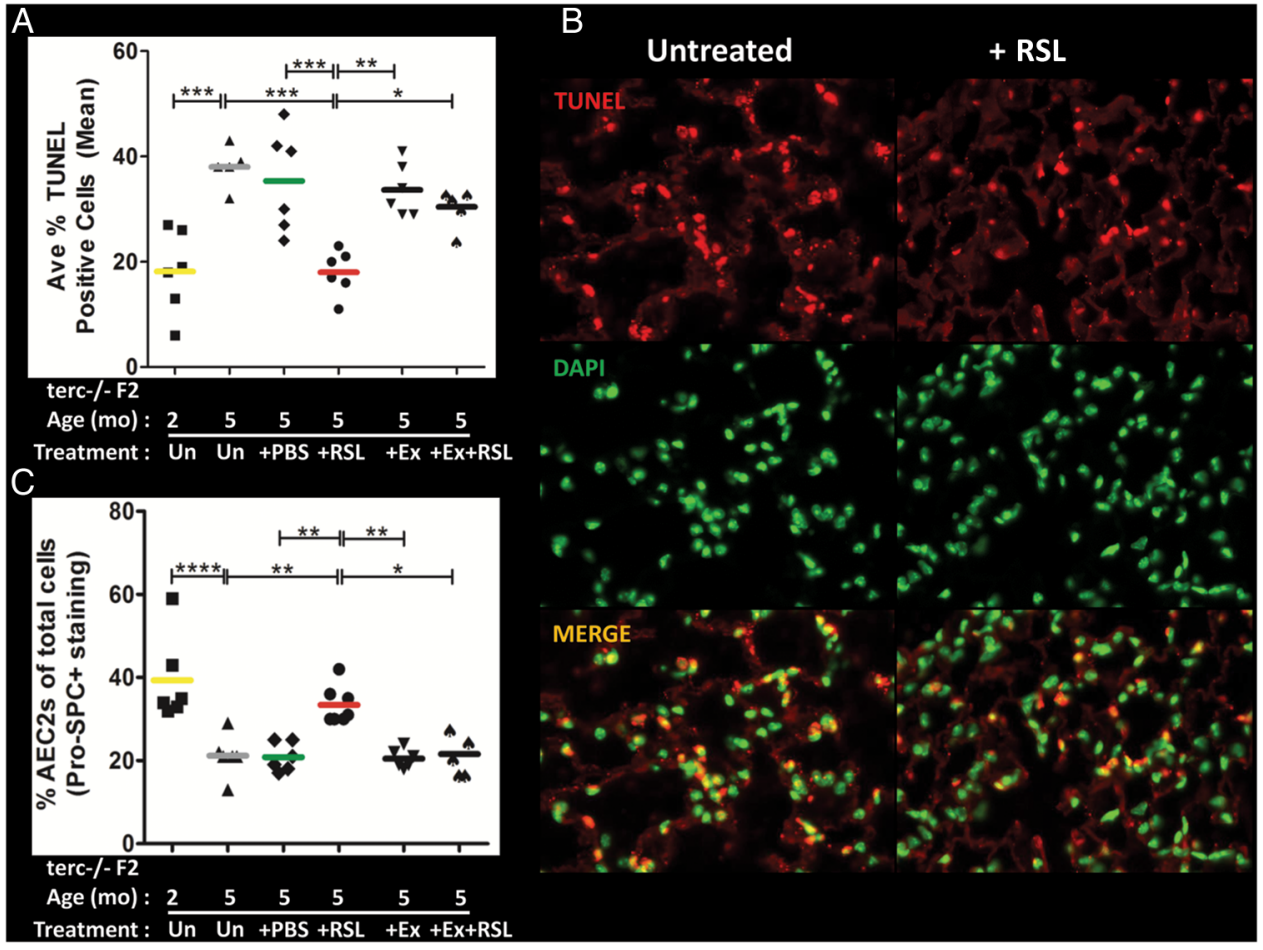

D

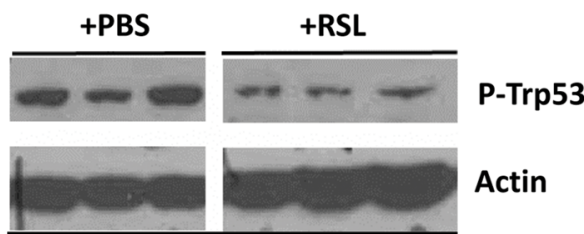

5months terc-/- F2 AEC2

E

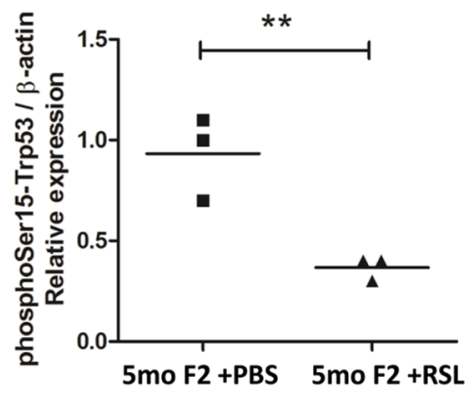

Figure 5 Percentage of TUNEL-positive cells in whole lung and p53 expression in alveolar epithelial type 2 cells (AEC2) following resveratrol (RSL) treatment. (A) Quantification of lung sections stained to detect cells carrying single-strand breaks in the lung parenchyma. By in situ detection with the TUNEL assay, cells containing DNA damage were identified and counted per lung section 1 month after the last RSL treatment or vehicle administration and also in untreated (Un) controls. TUNEL-positive cells were manually counted and are expressed as a mean per cent of the total number of nuclei per images per animal. Each data point corresponds to one animal and the group mean is indicated by the bar through each group. The data from the RSL-treated cohort show significantly lower numbers of TUNEL-positive cells compared with untreated and vehicle-administered controls aged 5 months $\left(\mathrm{N}=6,{ }^{* * *} \mathrm{p}<0.001\right)$, and compared with the Ex527 and Ex527 plus RSL controls aged 5 months $\left(\mathrm{N}=5-6,{ }^{* *} \mathrm{p}<0.001\right.$ and ${ }^{*} \mathrm{p}<0.05$. Statistical analysis was performed by one-way analysis of variance and the Bonferroni multiple comparison test. (B). Representative $40 \times$ images of TUNEL-stained lung sections from untreated terc-/-F2 mice aged 5 months and RSL-treated mice (terc-/-F2 vehicle-administered samples aged 2 months are not shown, but were similar to the untreated cohort). DNA damage was identified by TUNEL staining (red) and total nuclei by 4',6-diamidino-2-phenylindole (DAPI) staining (green). TUNEL-positive nuclei counted to generate quantitative data appear yellow in these pseudocoloured images due to overlay of red and green fluorescence. (C) Surfactant protein C (SPC) immunostaining was used to quantitate AEC2 numbers in lung sections. A reduction of AEC2 numbers was significant in untreated terc-I-F2 mice aged 2 and 5 months $(\mathrm{N}=6-7, * * * * \mathrm{p}<0.0001)$. Following RSL treatments, a significant increase in the SPC-positive cell numbers $\left({ }^{* *} \mathrm{p}<0.01\right)$ remained, while the AEC2 cell number remained significantly low in Ex527 and Ex527 plus RSL-treated terc-l-F2 mice $\left(\mathrm{N}=5-6,{ }^{* *} \mathrm{p}<0.01\right.$ and $\left.{ }^{*} \mathrm{p}<0.05\right)$. (D) Phospho-specific antibodies were used for immunoblotting lysates from isolated AEC2. RSL treatment led to reduction of pSer15-p53 in isolated terc-/-F2 AEC2 compared with levels in AEC2 isolated from the vehicle-administered cohort. Each lane represents AEC2 isolated from an individual mouse. Blotting for $\beta$-actin served as a protein loading control. (E) Student's t-test was used for analysis of quantitated band values generated by ImageJ densitometry $\left(\mathrm{N}=3\right.$ per group; $\left.{ }^{* *} \mathrm{p}<0.01\right)$. Data from an irrelevant cohort that was loaded between RSL-treated and phosphate buffered saline (PBS)-administered samples were excised from the blot image (vertical white space) in order to clarify the comparison. 


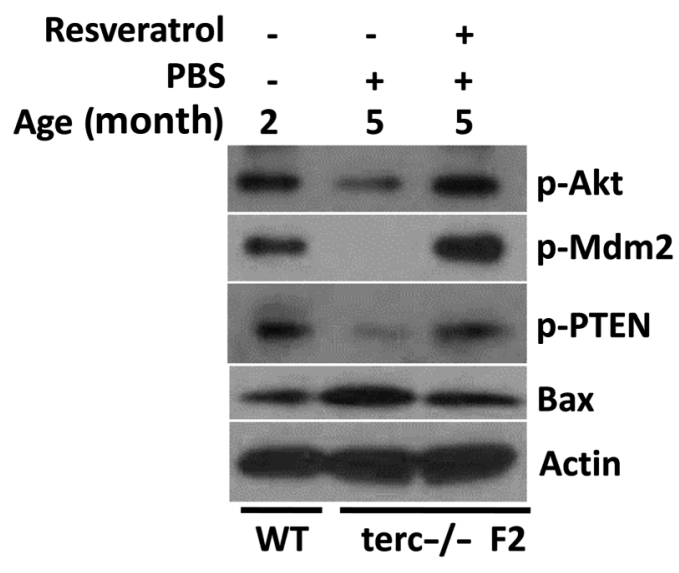

Figure 6 Expression of Akt survival pathway components in alveolar epithelial type 2 cells (AEC2) following resveratrol treatment. Phospho-specific antibodies were used for immunoblotting. Resveratrol treatment maintained expression of cell survival-related proteins in terc -I-F2 AEC2 compared with levels in AEC2 isolated from vehicle-administered cohorts. Resveratrol treatment maintained expression of cell survival-related proteins at levels observed in AEC2 isolated from wild type (WT) mice aged 2 months. Blot is representative of, at minimum, two blots. Each sample was composed of lysates equally pooled from three mice.
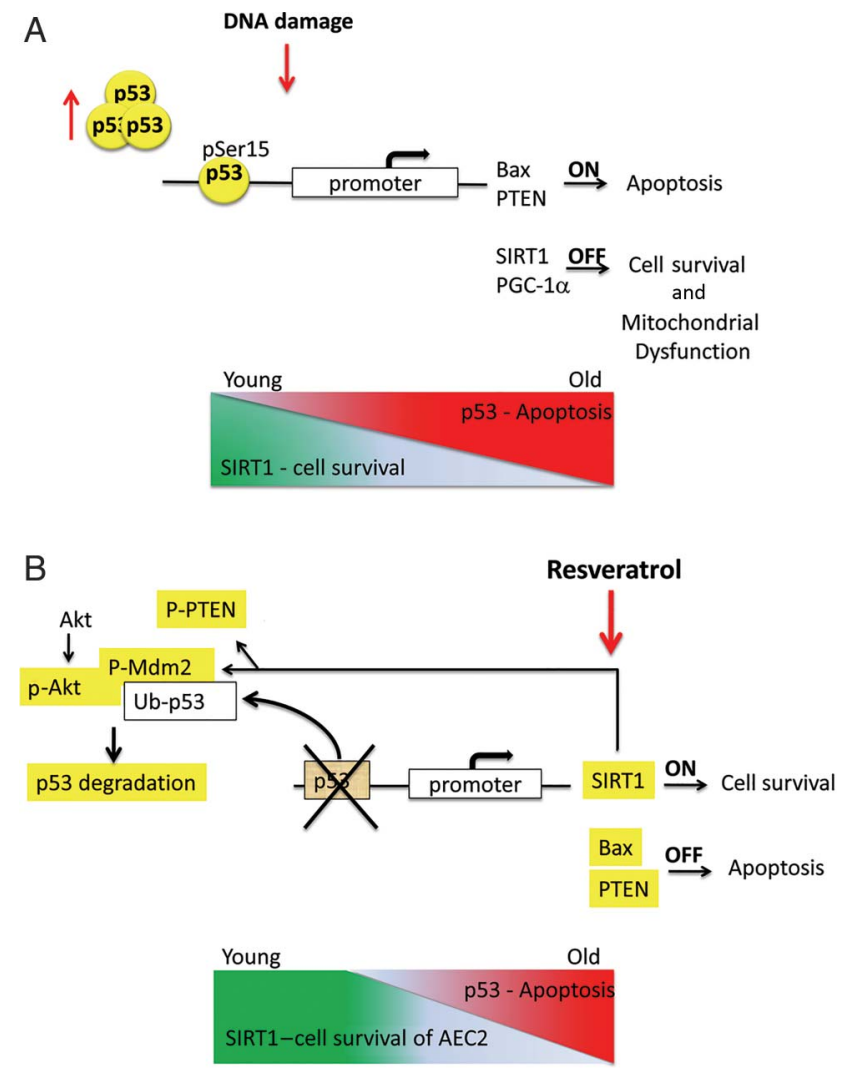

Figure 7 Proposed models for accelerated ageing in terc-/-F2 lung and the impact of resveratrol treatments. $(A)$ In the ageing lung alveolar epithelial type 2 cells (AEC2, stabilisation of p53 and accumulated damage lead to a raid shift towards an ageing phenotype, senescence and apoptosis. (B) Resveratrol treatments act directly to destabilise p53 and support the activity of survival pathways, which slows the shift towards p53 stabilisation and damage accumulation and results in maintenance of healthy $A E C 2$. that maintain it. RSL impact on other cellular compartments at this very early stage of ageing may be negligible due to the fact that our experiments show neither early ageing has a minimal impact on these cells, including lung immune cell populations, which means RSL has no true target in this time frame (data not shown). The impact of RSL treatment within the time frame of this study appears to be confined to AEC2 Trp53-regulated cell death pathways, the apoptotic response modulated by Bax, and Akt survival pathways. It does not appear to modulate AEC2 mitochondrial function. Rather, RSL prevents the loss of AEC2 by maintaining levels of cell survival proteins and protecting cells from death by suppression of DNA damage, which in turn contributes to maintenance of both lung function and structure. Taken together, these data show that RSL has the potential to act prophylactically on the AEC2 population before ageing-related breakdown occurs and thus potentially supports the AEC2 capacity for homeostatic repair that is necessary to maintain lung tissue integrity and function.

The proposed mechanism in our model of accelerated lung ageing (figure 7A) is that in response to DNA damage, including that induced by telomere shortening, $\operatorname{Trp} 53$ stabilises and accumulates at high levels. In youthful cells, Trp53 expression remains low and the protein undergoes constant turnover. Consequent to increased reactive oxygen species, DNA damage and/or ageing, Trp53 is stabilised and activated by phosphorylation of serine 15 along with other molecular changes. Stabilised p53 can then translocate to the nucleus to activate transcription of pro-apoptotic genes, including Bax and PTEN, and at the same time repress cell survival and mitochondrial genes, such as SIRT1 and PGC-1 $\alpha$. Ageing-related suppression of SIRT1 expression leads to $\operatorname{Trp} 53$ escaping deacetylation, which further supports its stabilisation and expression at high levels. Thus, activation of $\operatorname{Trp} 53$ by phosphorylation of serine 15 is a central signal that initiates cell ageing-related molecular signalling $^{35}$ and is a critical target for anti-ageing prophylaxis.

The proposed mechanism in our model, where RSL prophylaxis disrupts inevitable lung function decline (figure $7 \mathrm{~B}$ ), is maintenance of the Akt survival pathway in AEC2, deacetylation of $\operatorname{Trp} 53$ and activation of $\mathrm{Mdm} 2$ to sequester $\operatorname{Trp} 53$, all a consequence of SIRT1 induction. These events have all been mechanistically connected to diminished apoptosis and increased cellular survival. ${ }^{36} 37$ SIRT1 activity lies upstream of both these pathways and SIRT1-directed deacetylation and subsequent downregulation of Trp53 has been speculated to provide protection against ageing-related cellular apoptosis. This has been confirmed in our study by use of SIRT1 specific inhibitor Ex527, the use of which in IT deliveries, either alone or in combination with RSL, blunts the anti-apoptotic/pro-DNA damage repair impact of RSL and all other impacts of RSL that are downstream of SIRT1. These data show that SIRT1 activity is central to mediating the response to RSL.

Further obstruction of $\operatorname{Trp} 53$-directed apoptosis is provided by Thr308 phosphorylation of Akt, which phosphorylates the Trp53 binding protein MDM2 at serine 166/186. Once the Mdm2-Trp53 complex forms, $\operatorname{Trp} 53$ is ubiquitinated and degraded. As a result, there is downregulated expression of genes required for apoptosis and SIRT1 levels are further maintained during the treatment phase. The diminished levels of detectable TUNEL-positive cells in whole lung after RSL prophylaxis further supports modulation of SIRT1-Akt-Trp53dependent signalling to augment AEC2 stem cell survival and to alleviate the lung tissue changes associated with age-related lung deterioration. The apparent overall result of RSL inhalation is that the ageing process is slowed if administered early enough. 
This prophylactic approach helps preserve and maintain the function and structure of whole lung, presumably due to preservation of the adult stem cell AEC2 population, which then blocks further lung deterioration.

In agreement with the models presented in figure 7, we found that SIRT1 protein levels were indeed diminished in AEC2 isolated from terc-/-F2 vehicle-treated controls, but were elevated by RSL treatments, while no changes were observed in WT controls aged 2 and 5 months (data not shown). AEC2 PGC- $1 \alpha$ levels, which were diminished in untreated controls, were maintained by RSL, suggesting that PGC- $1 \alpha$ is also impaired, possibility downstream of a non-SIRT1 pathway. These findings linked inhaled RSL with mitochondria biology and, possibly, mitochondrial function. Although it has been reported that SIRT activation of PGC- $1 \alpha$ promotes mitochondrial biogenesis by improving energy deficits in other tissues, such as skeletal muscle, ${ }^{29}$ we found that inhaled RSL had no effect on mitochondrial respiration of AEC2 isolated from terc-/-F2 aged 5 months. Conversely, mitochondrial respiration was diminished in late-aged (16 months) terc-/-F2 AEC2 compared with early-aged (3 months) terc-/-F2 AEC2. It may be that AEC2 in terc-/-F2 mice aged 5 months have a higher ratio of undamaged (healthy) to damaged mitochondria despite diminishing PGC-1 $\alpha$ levels, resulting in net normal mitochondrial bioenergetics, and only in late-aged mice is a decline in mitochondrial bioenergetics noticeable. Indeed, this imbalance of unhealthy to healthy mitochondria has been speculated to underlie accelerated ageing and to create a context in which the ageing lung passes the point of successful functional rescue. Future studies could reveal this tipping point, which could have important implications for the efficacy of inhaled RSL as a practical, prophylactic treatment.

We have previously shown that the AEC2 population is heterogeneous in its response to injury, ${ }^{38}$ which suggests that distinct AEC2 subpopulations can be characterised by their capacity to respond to injury. We speculate that injury responsive AEC2 subpopulations diminish with age, thus progressively reducing the capacity of the lung to maintain homeostasis and drive repair. It may be that such subpopulations are particularly responsive to RSL treatments, which could potentially stimulate AEC2 re-entry into the cell cycle, thus replenishing viable AEC2 stem cell pools in the ageing lung. That being said, inhaled RSL treatments, while generally targeted to the lung but not specifically to AEC2, could also conceivably activate, directly or indirectly, other factors that support maintenance of the AEC2 stem cell population and inhibit AEC2 apoptosis, such as the endothelial cells of the capillary beds. Treatments could also maintain function of mesenchymal, immune and/or neuronal compartments, which were not directly examined in the current study.

We currently have no data to show if higher or lower RSL doses, delivered more or less frequently, could have a greater impact on maintaining distal lung function. The prophylactic protocol developed for our study differs from others in that we used a comparatively low RSL dose that was administered only three times. RSL studies in vitro used a dose range from micromolar to millimolar, while previous in vivo studies used much higher RSL doses delivered ad libidum in high fat diets. ${ }^{23} 2939$ One time, lung-specific RSL dosing has been tried for acute, experimental lung injury, ${ }^{40}$ but multiple doses used to address chronic lung conditions, such as ageing, have not previously been tried. We have no insight at present as to the long-term efficacy of low-dose, lung-targeted RSL prophylaxis, although we have observed that our standard treatment protocol does not have any significant impact on terc-/-F2 lifespan (data not shown). Questions regarding formulation, optimal dose, pharmacodynamics and target specificity of RSL prophylaxis will require further investigation.

\section{CONCLUSION}

RSL inhalational prophylaxis blocks ageing-related changes in expression of a coherent set of cell survival genes in AEC2, which strongly correlates with maintenance of more youthful lung structure and function. These findings suggest a potentially novel approach to maintaining lung health by preserving AEC2 integrity, prior to the irreversible onset of ageing-related structural and functional decline in the lungs.

Correction notice This article has been corrected since it was published Online First. The author list order has been corrected.

Acknowledgements We would like to thank Hemal H Mehta, the Seahorse Core Director at the USC Davis School of Gerontology, for assistance with the Seahorse assay. The authors also thank Sue Buckley for helpful discussions regarding the manuscript.

Contributors SN collaborated on study design, performed all experiments with the exception of western blotting, performed data analyses and cowrote the manuscript. RR performed western blots. JL performed mouse breeding and colony management. DW reviewed and revised the manuscript. BD conceived the project, collaborated on study design, performed data analysis and cowrote the manuscript.

Funding This work was supported by NIH/NHLBI R01 HL65352 and funding from the Pasadena Guild Endowment and the Garland Fund to BD and funding from the Pasadena Guild Endowment and the Garland Fund to DW. SN was supported by NIH/NIDCR T90 training grant DE021982.

Competing interests None declared.

Provenance and peer review Not commissioned; externally peer reviewed.

\section{REFERENCES}

1 Fletcher C, Peto R. The natural history of chronic airflow obstruction. $\mathrm{Br}$ Med J 1977;1:1645-8

2 Sharma G, Goodwin J. Effect of aging on respiratory system physiology and immunology. Clin Interv Aging 2006;1:253-60.

3 Wahba WM. Influence of aging on lung function-clinical significance of changes from age twenty. Anesth Analg 1983;62:764-76.

4 Imai K, Mercer BA, Schulman LL, et al. Correlation of lung surface area to apoptosis and proliferation in human emphysema. Eur Respir J 2005;25: 250-8.

5 Lowery EM, Brubaker AL, Kuhlmann E, et al. The aging lung. Clin Interv Aging 2013;8:1489-96.

6 Thannickal VJ, Murthy M, Balch WE, et al. Blue journal conference. Aging and susceptibility to lung disease. Am J Resp Crit Care 2015;191:261-9.

7 Yokohori N, Aoshiba K, Nagai A, et al. Increased levels of cell death and proliferation in alveolar wall cells in patients with pulmonary emphysema. Chest 2004;125:626-32.

8 Papaioannou Al, Rossios C, Kostikas K, et al. Can we delay the accelerated lung aging in COPD? Anti-aging molecules and interventions. Curr Drug Targets 2013;14:149-57.

9 Schouten LRA, Schultz MJ, van Kaam AH, et al. Association between maturation and aging and pulmonary responses in animal models of lung injury: a systematic review. Anesthesiology 2015;123:389-408.

10 Blasco MA, Lee HW, Hande MP, et al. Telomere shortening and tumor formation by mouse cells lacking telomerase RNA. Cell 1997;91:25-34.

11 Alder JK, Guo N, Kembou F, et al. Telomere length is a determinant of emphysema susceptibility. Am J Respir Crit Care Med 2011;184:904-12.

12 Barkauskas CE, Cronce MJ, Rackley CR, et al. Type 2 alveolar cells are stem cells in adult lung. J Clin Invest 2013;123:3025-36.

13 Jackson SR, Lee J, Reddy R, et al. Partial pneumonectomy of telomerase null mice carrying shortened telomeres initiates cell growth arrest resulting in a limited compensatory growth response. Am J Physiol Lung Cell Mol Physiol 2011;300: L898-909.

14 Lee J, Reddy R, Barsky L, et al. Lung alveolar integrity is compromised by telomere shortening in telomerase-null mice. Am J Physiol Lung Cell Mol Physiol 2009;296: L57-70.

15 Alder JK, Barkauskas CE, Limjunyawong N, et al. Telomere dysfunction causes alveolar stem cell failure. Proc Natl Acad Sci USA 2015;112: 5099-104. 
16 Harikumar KB, Aggarwal BB. Resveratrol: a multitargeted agent for age-associated chronic diseases. Cell Cycle 2008;7:1020-35.

17 Howitz KT, Bitterman KJ, Cohen HY, et al. Small molecule activators of sirtuins extend Saccharomyces cerevisiae lifespan. Nature 2003:425:191-6.

18 Houtkooper RH, Pirinen E, Auwerx J. Sirtuins as regulators of metabolism and healthspan. Nat Rev Mol Cell Bio 2012;13:225-38.

19 Chang HC, Guarente L. SIRT1 and other sirtuins in metabolism. Trends Endocrin Met 2014;25:138-45.

20 Hori YS, Kuno A, Hosoda R, et al. Regulation of FOXOs and p53 by SIRT1 modulators under oxidative stress. PLOS ONE 2013;8:e73875.

21 Lee JT, Gu W. SIRT1: regulator of p53 deacetylation. Genes Cancer 2013:4:112-17.

22 Imboden M, Schwartz J, Schindler C, et al. Decreased PM10 exposure attenuates age-related lung function decline: genetic variants in p53, p21, and CCND1 modify this effect. Environ Health Persp 2009;117:1420-7.

23 Price NL, Gomes AP, Ling AJ, et al. SIRT1 is required for AMPK activation and the beneficial effects of resveratrol on mitochondrial function. Cell Metab 2012;15:675-90.

24 Handschin C, Spiegelman BM. Peroxisome proliferator-activated receptor $\gamma$ coactivator 1 coactivators, energy homeostasis, and metabolism. Endocr Rev 2006;27:728-35

25 Rajendrasozhan S, Yang SR, Kinnula VL, et al. SIRT1, an anti-inflammatory and antiaging protein, is decreased in lungs of patients with chronic obstructive pulmonary disease. Am J Respir Crit Care Med 2008;177:861-70.

26 Timmers $S$, Auwerx J, Schrauwen P. The journey of resveratrol from yeast to human Aging (Albany NY) 2012:4:146-58.

27 Chung SW, Yao HW, Caito S, et al. Regulation of SIRT1 in cellular functions: role of polyphenols. Arch Biochem Biophys 2010;501:79-90.
28 Baur JA, Sinclair DA. Therapeutic potential of resveratrol: the in vivo evidence. Nat Rev Drug Discov 2006;5:493-506.

29 Lagouge M, Argmann C, Gerhart-Hines Z, et al. Resveratrol improves mitochondrial function and protects against metabolic disease by activating SIRT1 and PGC-1alpha. Cell 2006;127:1109-22.

30 Feige JN, Lagouge M, Canto $C$, et al. Specific SIRT1 activation mimics low energy levels and protects against diet-induced metabolic disorders by enhancing fat oxidation. Cell Metab 2008:8:347-58.

31 Hubbard BP, Sinclair DA. Small molecule SIRT1 activators for the treatment of aging and age-related diseases. Trends Pharmaco/ Sci 2014;35:146-54.

32 Park SJ, Ahmad F, Philp A, et al. Resveratrol ameliorates aging-related metabolic phenotypes by inhibiting cAMP phosphodiesterases. Cell 2012;148:421-33.

33 Van Houten B, Woshner V, Santos JH. Role of mitochondrial DNA in toxic responses to oxidative stress. DNA Repair (Amst) 2006;5:145-52.

34 Scarpulla RC. Transcriptional paradigms in mammalian mitochondrial biogenesis and function. Physiol Rev 2008;88:611-38

35 Samuels-Lev Y, O'Connor DJ, Bergamaschi D, et al. ASPP proteins specifically stimulate the apoptotic function of p53. Mol Cell 2001;8:781-94.

36 Luo J, Nikolaev AY, Imai S, et al. Negative control of p53 by Sir2 $\alpha$ promotes cell survival under stress. Cell 2001;107:137-48.

37 Vaziri H, Dessain SK, Ng Eaton E, et al. hSIR2(SIRT1) functions as an NAD-dependent p53 deacetylase. Cell 2001;107:149-59.

38 Reddy R, Buckley S, Doerken M, et al. Isolation of a putative progenitor subpopulation of alveolar epithelial type 2 cells. Am J Physiol Lung Cell Mol Physiol 2004;286:L658-67.

39 Baur JA, Pearson KJ, Price NL, et al. Resveratrol improves health and survival of mice on a high-calorie diet. Nature 2006;444:337-42.

40 Cao Q, Jing C, Tang $X$, et al. Protective effect of resveratrol on acute lung injury induced by lipopolysaccharide in mice. Anat Rec (Hoboken) 2011;294:527-32. 